\title{
Nature and Mediators of Parietal Epithelial Cell Activation in Glomerulonephritides of Human and Rat
}

\author{
Paola Rizzo, ${ }^{*}$ Norberto Perico, ${ }^{*}$ Elena Gagliardini, ${ }^{*}$ Rubina Novelli, ${ }^{*}$ Malcolm R. Alison, ${ }^{\dagger}$ Giuseppe Remuzzi, ${ }^{\star}$ and \\ Ariela Benigni*
}

\begin{abstract}
From the Mario Negri Institute for Pharmacological Research (IRCCS), * Centro Anna Maria Astori, Science and Technology Park Kilometro Rosso, Bergamo, Italy; the Centre for Tumour Biology, ${ }^{\dagger}$ Barts Cancer Institute, Barts and The London School of Medicine and Dentistry, London, United Kingdom; and the Unit of Nephrology and Dialysis, ${ }^{\ddagger}$ Azienda Ospedaliera Papa Giovanni XXIII, Bergamo, Italy
\end{abstract}

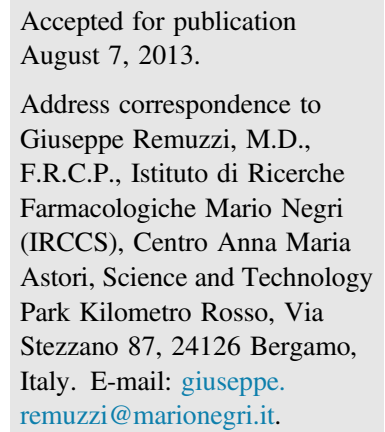

\begin{abstract}
Bowman's capsule parietal epithelial cell activation occurs in several human proliferative glomerulonephritides. The cellular composition of the resulting hyperplastic lesions is controversial, although a population of $\mathrm{CD} 133^{+} \mathrm{CD} 24^{+}$progenitor cells has been proposed to be a major constituent. Mediator(s) involved in proliferation and migration of progenitor cells into the Bowman's space have been poorly explored. In a series of 36 renal biopsies of patients with proliferative and nonproliferative glomerulopathies, dysregulated $\mathrm{CD} 133^{+} \mathrm{CD} 24^{+}$progenitor cells of the Bowman's capsule invade the glomerular tuft exclusively in proliferative disorders. Up-regulation of the CXCR4 chemokine receptor on progenitor cells was accompanied by high expression of its ligand, SDF-1, in podocytes. Parietal epithelial cell proliferation might be sustained by increased expression of the angiotensin II (Ang II) type-1 (AT ${ }_{1}$ ) receptor. Similar changes of CXCR4, SDF-1, and $\mathrm{AT}_{1}$ receptor expression were found in Munich Wistar Frömter rats with proliferative glomerulonephritis. Moreover, an angiotensin-converting enzyme inhibitor normalized CXCR4 and $\mathrm{AT}_{1}$ receptor expression on progenitors concomitant with regression of crescentic lesions in a patient with crescentic glomerulonephritis. These results suggest that glomerular hyperplastic lesions derive from the proliferation and migration of renal progenitors in response to injured podocytes. The Ang II/AT 1 receptor pathway may participate, together with SDF-1/CXCR4 axis, to the dysregulated response of renal precursors. Thus, targeting the Ang II/AT 1 receptor/CXCR4 pathways may be beneficial in severe forms of glomerular proliferative disorders. (Am J Pathol 2013, 183: 1769-1778; http://dx.doi.org/10.1016/j.ajpath.2013.08.008)
\end{abstract}

Glomerular injury caused by multiple etiologies can lead to activation and accumulation of parietal epithelial cells within the Bowman's space as a common response to damage. ${ }^{1}$ The most diffuse lesions are found in rapidly progressive idiopathic glomerulonephritis, Wegener's granulomatosis, and anti-glomerular basement membrane antibody disease. Extracapillary proliferation can also be observed in lupus nephritis, IgA nephropathy, and membranoproliferative glomerulonephritis, ${ }^{2,3}$ and if left untreated, can result in rapidly progressive renal failure. ${ }^{4}$

Understanding the mechanism(s) for the formation of these multilayered cellular lesions would lead to the development of more targeted therapies than the currently used cytotoxic agents. Although extracapillary proliferation is a relatively straightforward pathological change to recognize, more controversial has been determining its cellular components and their possible pathogenic role. The traditional concepts have come largely from immunohistochemical studies that have concluded that multilayered cellular lesions are a mixture of glomerular parietal epithelial cells, macrophages, and myofibroblasts, ${ }^{5-7}$ the proportion of such cells in the lesion being variable. In both animal models and human tissues, parietal epithelial cells predominate when Bowman's capsule is intact, whereas macrophages and myofibroblasts

\footnotetext{
Supported in part by a grant from Ministero della Salute, Bando Cellule Staminali 2008 (Codice Progetto B11J11001110002) and by a fellowship from Fondazione Aiuti per la Ricerca sulle Malattie Rare (ARMR), Bergamo, Italy (PR). This work was partially supported by a European Commission grant, project STELLAR n ${ }^{\circ}$ HEALTH-F4-2012-305436.

P.R. and N.P. contributed equally to this work.
} 
prevail when Bowman's capsule is ruptured, ${ }^{5,8,9}$ an event reported to be rare. ${ }^{10}$ More recent studies also suggested the podocytes as key constituents of the crescents, ${ }^{11-14}$ but their role remains unclear. Given their terminally differentiated phenotype, ${ }^{15-17}$ they could not theoretically migrate and proliferate, limiting their contribution, if any, in extracapillary lesion formation. Recently, a heterogeneous population of renal progenitor cells, previously identified in normal human Bowman's capsule, ${ }^{18}$ has been documented in hyperplastic lesions of human crescentic glomerulonephritis. ${ }^{19}$ It has been suggested that these extracapillary lesions could be the result of dysregulated proliferation of renal progenitor cells in response to the injured podocytes. ${ }^{19}$ This possibility is supported by our finding in Munich Wistar Frömter (MWF) rats, which are genetically programed to develop renal damage characterized by excessive progenitor cell migration and proliferation leading to their accumulation into cellular lesions and glomerulosclerosis. ${ }^{20}$ Nevertheless, the mechanisms and mediators responsible for the intraglomerular accumulation of renal progenitor cells in proliferative glomerulonephritis remain ill-defined. Evidence in SCID mice with acute renal failure indicates an important role of stromal cell-derived factor-1 (SDF-1) and its receptor C-X-C chemokine receptor type 4 (CXCR4) in the therapeutic migration of renal progenitor cells. ${ }^{21}$ Because activation of the angiotensin II (Ang II)/angiotensin II type-1 $\left(\mathrm{AT}_{1}\right)$ receptor pathway may contribute to cell proliferation and migration processes, ${ }^{22}$ and based on previous findings that angiotensin-converting enzyme (ACE) inhibition reduced glomerular lesions by limiting renal progenitor cell migration, ${ }^{20}$ we reasoned that the $\mathrm{AT}_{1}$ receptor could also play a key role in the abnormal proliferation of renal progenitors underlying the hyperplastic lesions.

Therefore, building on the previous experimental work suggesting that both the SDF-1/CXCR4 and the Ang II/AT receptor pathway play a role in sustaining the migratory/ proliferative properties of renal progenitor cells, we sought to get further insights into the cellular mechanism(s) and mediators contributing to extracapillary proliferation in humans. In particular, our efforts focused on i) further highlighting the key role of renal progenitor cells in the multilayered accumulation of proliferating cells in the Bowman's space of patients with proliferative glomerulonephritis; ii) dissecting the contribution of the SDF-1/CXCR4 axis to the migratory property of dysregulated progenitor cells; and iii) testing whether the Ang II/AT 1 receptor pathway critically participates, together with the chemokine/receptor, to migration and proliferation of renal progenitors, resulting in the progression of extracapillary lesions.

\section{Materials and Methods}

\section{Patients}

Thirty six patients with proliferative and nonproliferative glomerulopathies from the archives of the Unit of
Nephrology, Azienda Ospedaliera Papa Giovanni XXIII, Bergamo, Italy, were enrolled in the study. Patients with glomerular extracapillary proliferative lesions had either extracapillary glomerulonephritis $(n=9)$ or IgA nephropathy $(n=9)$. Patients with nonproliferative diseases were diagnosed as either membranous nephropathy $(n=7)$ or diabetic nephropathy $(n=11)$. Written informed consent was obtained from all these patients. Demographic, clinical, and hematochemical parameters at the time of renal biopsy were retrieved from the hospital database. In addition, to further address the possible participation of the Ang II/AT 1 receptor pathway and renal progenitor cells in the development of glomerular hyperplastic lesions, we analyzed in-depth renal tissue specimens from one of these patients with antineutrophil cytoplasm antibody-positive crescentic glomerulonephritis from whom two biopsies were collected, before and after 8 months therapy with the ACE inhibitor ramipril (titrating up the dose from 2.5 to $7.5 \mathrm{mg} /$ day) associated with the immunosuppressant azathioprine $(50 \mathrm{mg} /$ day $)$. All kidney biopsy specimens considered for the present study had been originally obtained for the diagnosis of renal disease. In addition to specimens from patients with proliferative and nonproliferative diseases, renal biopsies from an uninvolved portion of kidney collected from tumor nephrectomy specimens were obtained from 10 patients and used as controls.

\section{Immunofluorescence and Confocal Microscopy}

Archived kidney biopsies, snap frozen in liquid nitrogen and embedded in OCT compound, were used for immunofluorescence analysis. Double or triple immunostaining for CD24, CD133, CXCR4, SDF-1, nephrin, CD68, ED1, and $\mathrm{AT}_{1}$ receptor expression was performed. Three-micron frozen sections were air dried, fixed with cold acetone, washed with PBS, and incubated with $1 \%$ bovine serum albumin to block nonspecific sites. The following primary antibodies were used: goat or mouse anti-CD24 (1:25; Santa Cruz Biotechnology, Santa Cruz, CA), mouse anti-CD133 (1:50; Miltenyi Biotec, Bergisch Gladbach, Germany), rabbit anti-CXCR4 (1:50; Abcam, Cambridge, UK), rabbit anti-SDF-1 (1:200; Abcam), goat antinephrin (1:50; Santa Cruz Biotechnology), mouse anti-CD68 (1:100; Dako, Glostrup, Denmark), mouse anti-ED1 (1:100; Millipore, Billerica, MA) and rabbit anti- $\mathrm{AT}_{1}$ receptor (1:25; Santa Cruz Biotechnology), followed by the specific fluorescein isothiocyanate or Cy3-conjugated secondary antibodies (Jackson ImmunoResearch Laboratories, West Grove, PA). Nuclei were stained with DAPI, and the renal structure with lectins, either rhodamine lens culinaris agglutinin or fluorescein wheat germ agglutinin (Vector Laboratories, Burlingame, CA). Negative controls were obtained by omitting primary antibodies on adjacent sections. Fluorescence was examined by an inverted confocal laser scanning microscope (LS 510 Meta; Zeiss, Jena, Germany). For SDF-1 expression in patients with extracapillary glomerulonephritis and in normal kidneys, all glomeruli were acquired and subjected to semiquantitative analysis. Glomerular SDF-1 expression 
was graded on a scale of 0 to 3 (0: no staining, 1: mild, 2: moderate, 3 : strong diffuse). For $\mathrm{AT}_{1}$ receptor expression in MWF and Wistar rats, all glomeruli were acquired and subjected to quantitative analysis. The number of $\mathrm{AT}_{1}$ receptor $^{+}$ cells was counted with respect to the Bowman's capsule length.

\section{Immunoperoxidase}

Human biopsies, which contained 5 to 30 glomeruli, were also analyzed for $\mathrm{AT}_{1}$ receptor expression by immunoperoxidase staining. Duboscq-Brazil-fixed, 3- $\mu \mathrm{m}$ paraffinembedded kidney sections were deparaffinized, rehydrated, and incubated for 30 minutes with $0.3 \% \mathrm{H}_{2} \mathrm{O}_{2}$ in methanol to quench endogenous peroxidase. Antigen retrieval was performed using microwave [twice for 5 minutes in citrate buffer $10 \mathrm{mmol} / \mathrm{L}$ (pH 6.0) at an operating frequency of $2450 \mathrm{MHz}$ and $600 \mathrm{~W}$ power output] and citrate buffer incubation (15 minutes at room temperature) to increase the reactivity of the antibody to antigen. After blocking with $1 \%$ bovine serum albumin, sections were incubated with rabbit anti-AT $\mathrm{T}_{1}$ receptor primary antibody (1:25; Santa Cruz Biotechnology), followed by a specific biotinylated secondary antibody (Jackson ImmunoResearch Laboratories) and diaminobenzidine (Merck, Darmstadt, Germany) substrate solution. Slides were finally counterstained with hematoxylin, dehydrated in graded alcohols, mounted with coverslips, and then observed by light microscopy (ApoTome Axio Imager Z2; Zeiss). Negative controls were obtained by omitting the primary antibody on adjacent sections.

\section{MWF Rat Model of Crescentic Glomerulonephritis}

Eighteen male MWF rats from our colony that develop renal damage characterized by extracapillary lesions and glomerulosclerosis ${ }^{20}$ were divided into two groups as follows: group $1(n=6)$ received saline and was sacrificed at 60 weeks of age; group $2(n=6)$ received the ACE inhibitor lisinopril $(80 \mathrm{mg} / \mathrm{L}$ in the drinking water) from 50 to 60 weeks of age. Sixty-week-old Wistar rats (Charles River S.p.A., Calco, Italy) were used as controls $(n=6)$. All rats were maintained in a room with constant temperature and light, having free access to water and food. Animal care and treatment were conducted according to the institutional guidelines that are compliant with national (Decreto Legislativo n116, Gazzetta Ufficiale suppl. 40, 18/2/1992, Circolare N.8, Gazzetta Ufficiale 14/7/1994) and international laws and policies (EEC Council Directive 86/609, OJL 358-1, 1987; Guide for the Care and Use of Laboratory Animals. NIH publication n. 85-23. Revised 1996). All animal studies were approved by the Institutional Animal Care and Use Committees of IRCCS - Istituto di Ricerche Farmacologiche Mario Negri. At sacrifice, kidneys were perfused with PBS under anesthesia, fixed in $4 \%$ paraformaldehyde, snap frozen in liquid nitrogen, and embedded in optimal cutting temperature compound.

\section{Statistical Analysis}

Results were expressed as means \pm SD. Statistical analysis of $\mathrm{AT}_{1}$ receptor quantification in experimental animals was performed using analysis of variance with the Bonferroni post hoc analysis for multiple comparisons. The Student's $t$-test was applied for SDF-1 quantification in patients. Statistical significance was defined as $P<0.05$.

\section{Results}

\section{Clinical and Histopathological Characteristics of Patient Populations}

Two cohorts of patients affected by glomerular diseases associated with or without extracapillary proliferation at renal biopsy were evaluated, and their clinical and histopathological characteristics are outlined in Table 1. Demographic and clinical findings at the time of biopsy were similar among the different groups. The mean age of the patients was 53 years, and there was a slight male predominance. There were no differences in baseline systolic and diastolic blood pressure among groups, whereas proteinuria and serum creatinine levels were slightly lower in patients with $\operatorname{Ig} \mathrm{A}$ nephropathy. At histological examination, renal biopsies of patients with extracapillary glomerulonephritis, and to a lesser extent with IgA nephropathy, showed a high percentage of glomeruli with extracapillary proliferation. These proliferative lesions were absent in biopsies of patients with membranous or diabetic nephropathies.

\section{$\mathrm{CD}_{133^{+}} \mathrm{CD}_{24}{ }^{+}$Renal Progenitor Cells Are Major Constituents of Hyperplastic Lesions}

Consistent with our previous study, ${ }^{19}$ multiple layers of cells in the Bowman's space of tissue specimens from patients with extracapillary glomerulonephritis were mostly composed of $\mathrm{CD} 133^{+} \mathrm{CD} 24^{+}$cells (Figure 1A). To further confirm that accumulation of progenitor cells in the glomerular tuft was a feature of proliferative diseases, we also analyzed biopsies of patients with IgA nephropathy, in which abundant $\mathrm{CD} 133^{+} \mathrm{CD} 24^{+}$cells were consistently found in all segmental lesions between the Bowman's capsule and the glomerular capillary tuft (Figure 1B). Exuberant progenitor cell accumulation was confined to proliferative disorders, as it was not found in biopsies of patients with membranous nephropathy (Figure 1C) and diabetic nephropathy (Figure 1D). In these latter settings, the distribution of $\mathrm{CD} 133^{+} \mathrm{CD} 24^{+}$ cells along the Bowman's capsule was comparable to control kidneys (Figure 1E).

\section{CXCR4 Is Overexpressed in Progenitor Cells within Extracapillary Lesions}

In vitro, human renal progenitor cells express the chemokine receptor CXCR4, which has been related to the migratory 
Table 1 Demographic, Clinical, and Histopathological Characteristics of Patient Populations

\begin{tabular}{|c|c|c|c|c|}
\hline & \multicolumn{2}{|l|}{ Proliferative } & \multicolumn{2}{|c|}{ Nonproliferative } \\
\hline & $\begin{array}{l}\text { Extracapillary } \\
\text { glomerulonephritis }\end{array}$ & IgA nephropathy & $\begin{array}{l}\text { Membranous } \\
\text { nephropathy }\end{array}$ & $\begin{array}{l}\text { Diabetic } \\
\text { nephropathy }\end{array}$ \\
\hline Age, years & $32-81$ & $32-50$ & $44-66$ & $54-70$ \\
\hline Male:female & $3: 6$ & $7: 1$ & $4: 3$ & $9: 1$ \\
\hline $\mathrm{SBP}, \mathrm{mm} \mathrm{Hg}$ & $135.6 \pm 4.7$ & $126.0 \pm 8.1$ & $136.8 \pm 4.7$ & $145.8 \pm 5.4$ \\
\hline Serum creatinine, $\mathrm{mg} / \mathrm{dL}$ & $3.9 \pm 0.9$ & $1.8 \pm 0.6$ & $2.5 \pm 0.6$ & $1.2 \pm 0.2$ \\
\hline Glomeruli with extracapillary proliferation, $\%$ & $60.9 \pm 8.6$ & $15.0 \pm 5.8$ & $0.0 \pm 0.0$ & $0.4 \pm 0.0$ \\
\hline Normal glomeruli, \% & $9.4 \pm 5.5$ & $16.1 \pm 5.6$ & $17.9 \pm 4.6$ & $30 \pm 7.2$ \\
\hline Glomeruli with global sclerosis, \% & $17.5 \pm 4.3$ & $32.8 \pm 9.2$ & $14.8 \pm 7.9$ & $15.0 \pm 3.8$ \\
\hline Glomeruli with segmental disorders, $\%$ & $4.4 \pm 3.0$ & $14.4 \pm 3.4$ & $1.9 \pm 1.9$ & $27.2 \pm 10.2$ \\
\hline
\end{tabular}

${ }^{*}$ Capillary loop thickening, mesangial hypercellularity and matrix expansion, and epithelial vacuolization.

DBP, diastolic blood pressure; SBP, systolic blood pressure.

property of these cells. ${ }^{21}$ We first evaluated the expression of CXCR4 in renal progenitor cells in proliferative glomerulonephritis and nonproliferative glomerular disorders. Biopsy immunostaining of patients with extracapillary glomerulonephritis and IgA nephropathy revealed high CXCR4 expression on the majority of $\mathrm{CD} 24^{+}$progenitor cells within hyperplastic lesions (Figure 2A). On the contrary, in biopsies from patients with membranous nephropathy or diabetes (Figure 2, B and C), the expression of CXCR4 was faint and mainly localized in the Bowman's capsule, similar to the distribution in glomeruli from normal kidneys, where staining was restricted to a few CD $24^{+}$progenitor cells (Figure 2D).

\section{Podocytes Express the CXCR4 Ligand, SDF-1}

Concomitant with high expression of CXCR4 in renal progenitor cells, a significantly increased expression of the
CXCR4 ligand, SDF-1, was found in glomerular tuft of biopsies from patients with proliferative glomerulonephritis in respect to controls (Figure 3, A-C). Quantification of SDF-1 revealed that the difference was statistically significant $(P<$ 0.05) (Figure 3D). By double immunostaining, we investigated the cell population that expressed SDF-1. Macrophages were found to infiltrate the hyperplastic lesions as revealed by the presence of $\mathrm{CD}^{+} 8^{+}$cells in hyperplastic lesions (Figure 3A). Double immunostaining of CD68 and SDF-1 revealed that macrophages were not the cellular source of SDF-1 (Figure 3A) as documented by the absence of signal colocalization. Instead, co-staining of nephrin and SDF-1 revealed that podocytes expressed SDF-1 and so, presumably, provide the ligand for CXCR4 in patients with proliferative glomerulonephritis (Figure 3B). A few parietal epithelial cells also expressed SDF-1 to a comparable extent in proliferative diseases and in controls (Figure 3, A-C).
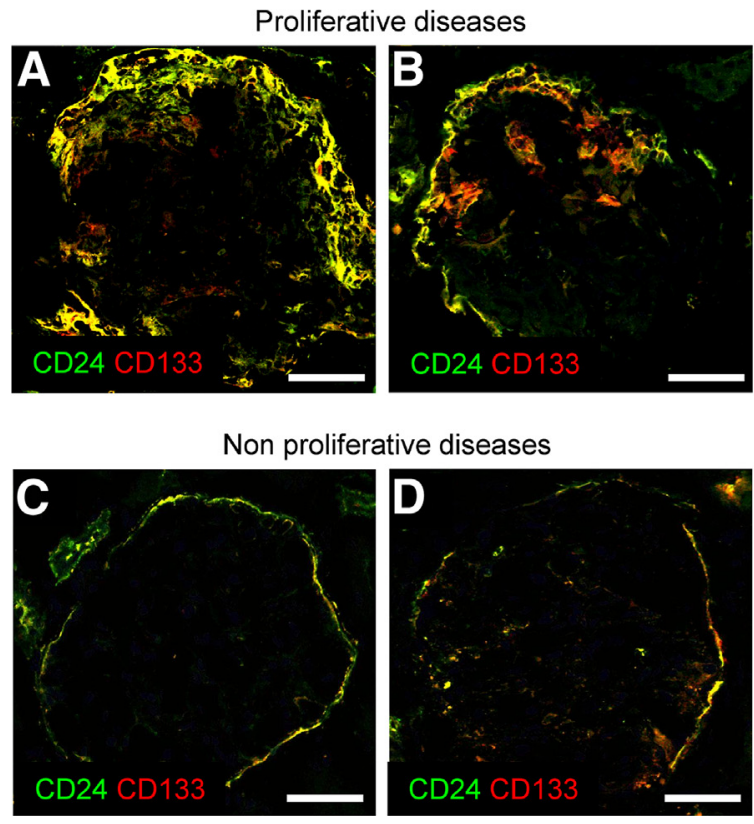

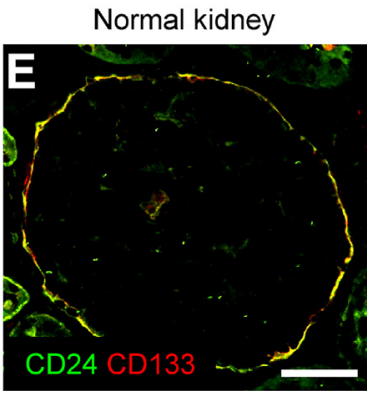

Figure 1 CD133-CD24 expression in patients with proliferative and nonproliferative disorders. A: Double immunofluorescence staining for CD24 (green) and CD133 (red) revealed that in a glomerulus of a patient with extracapillary glomerulonephritis, the two antigens colocalized inside crescentic lesions. B: In IgA nephropathy, abundant $\mathrm{CD} 133^{+} \mathrm{CD}_{2} 4^{+}$cells are found in the lesions between the Bowman's capsule and the glomerular capillary tuft. C-E: In membranous nephropathy (C) and in diabetic nephropathy (D), $\mathrm{CD} 133^{+} \mathrm{CD} 24^{+}$ cells are confined to the Bowman's capsule as observed in normal control kidneys (E). Scale bars: $50 \mu \mathrm{m}$. 


\section{Proliferative diseases}
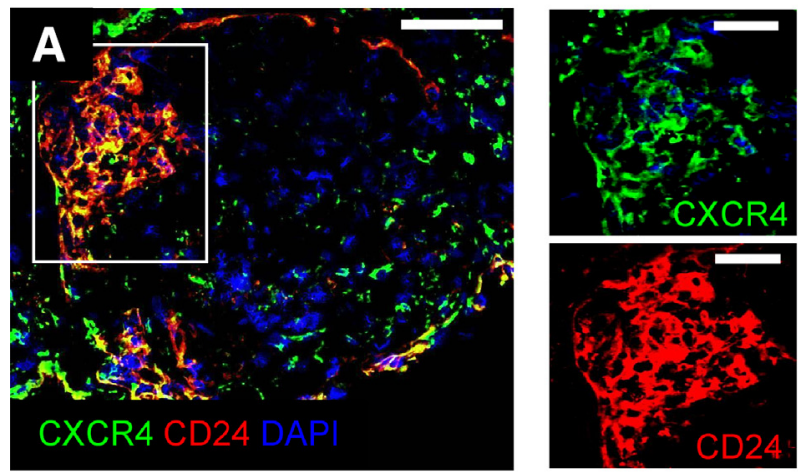

Non proliferative diseases
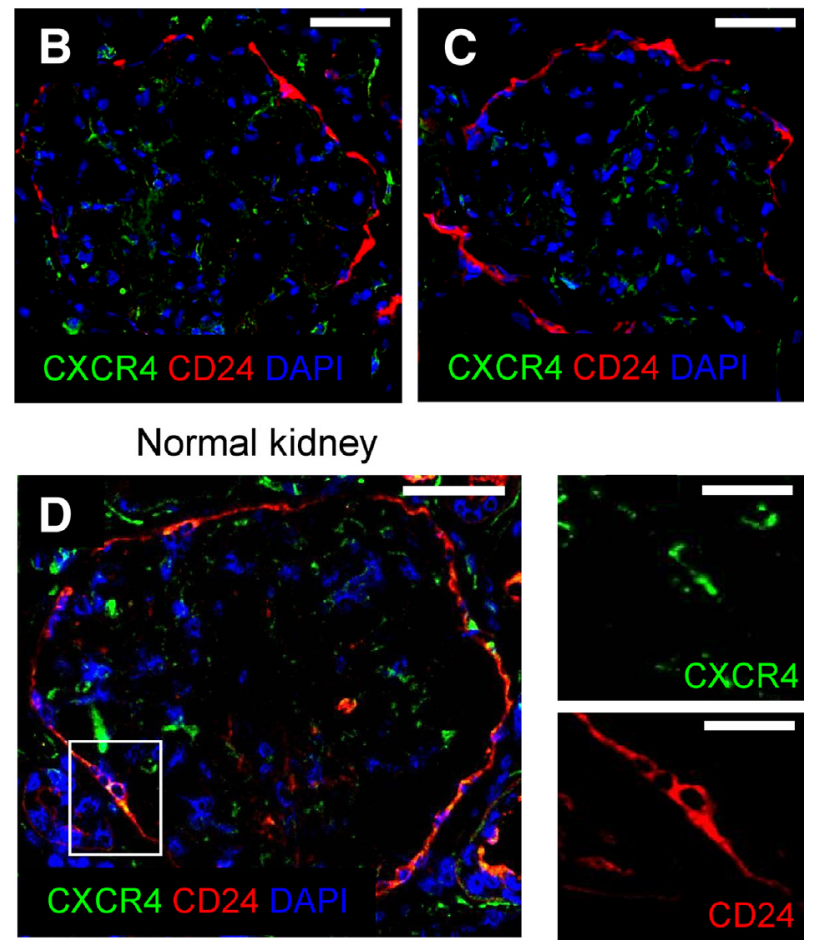

Figure 2 CXCR4 is overexpressed in progenitor cells within hyperplastic lesions in patients with proliferative diseases. A: In patients with extracapillary glomerulonephritis, CXCR4 (green) is expressed by the majority of $\mathrm{CD} 24^{+}$progenitor cells (red) within hyperplastic lesions. High magnification of CXCR4 and CD24 staining (inset) is shown in the right panels. B and C: In patients with membranous (B) and diabetic (C) nephropathies, CXCR4 expression is faint, and no cells in the Bowman's capsule coexpressed the CD24 progenitor marker. D: Representative photomicrograph of double staining for CXCR4 and CD24 in a normal human glomerulus showing that few cells in the Bowman's capsule co-expressed the two markers. High magnification of CXCR4 and CD24 staining in the Bowman's capsule (inset) is seen in the right panels. DAPI (blue) stained nuclei. Scale bars: $50 \mu \mathrm{m} ; 25 \mu \mathrm{m}$ for high magnification (A and $\mathbf{D}$, right panels).

\section{$\mathrm{AT}_{1}$ Receptor Overexpression on Progenitor Cells in Human Proliferative Disorders}

Because activation of the Ang II/AT 1 receptor pathway may favor cell proliferation and migration, ${ }^{22}$ we then assessed the expression of the $\mathrm{AT}_{1}$ receptor in progenitor cells in proliferative disorders. In both patients with extracapillary glomerulonephritis and IgA nephropathy, the $\mathrm{AT}_{1}$ receptor was abundantly expressed by progenitor cells along the Bowman's capsule as well as within cellular synechiae, the early morphological abnormalities preceding extracapillary lesion formation ${ }^{20}$ (Figure 4A). Expression of the $\mathrm{AT}_{1}$ receptor was maintained by progenitor cells forming crescentic lesions, as demonstrated by double immunofluorescence staining with the progenitor cell marker CD24 (Figure 4B). This was not the case in biopsy tissues from patients with membranous nephropathy and diabetes (Figure 4C) and in normal kidney (Figure 4D), where $\mathrm{AT}_{1}$ receptor expression was only confined to a few cells in the Bowman's capsule (Figure 4, C and D). Colocalization of the $\mathrm{AT}_{1}$ receptor with $\mathrm{CD} 24$ in normal kidney indicated that the rare parietal epithelial cells expressing this receptor were progenitor cells (Figure 4E).

\section{Pattern of CXCR4 and SDF-1 Expression in Hyperplastic Lesions in the Rat}

To evaluate whether parietal epithelial cell activation translated to more CXCR4 expression as in patients with proliferative glomerulonephritides, we studied the MWF rat model characterized by extracapillary proliferation and glomerulosclerosis. ${ }^{20}$ Consistent with the human results, CXCR4 expression was markedly increased in the hyperplastic lesions of MWF rats (Figure 5A). The CXCR4 ligand SDF-1 was localized to podocytes in MWF rats as documented by double immunostaining for the chemokine and nephrin (Figure 5D). By contrast, SDF-1 staining showed no colocalization with ED1 macrophages (Figure 5G). MWF rats receiving ACE inhibitor treatment showed a normalization of the immunostaining for both CXCR4 and SDF-1 (Figure 5, B
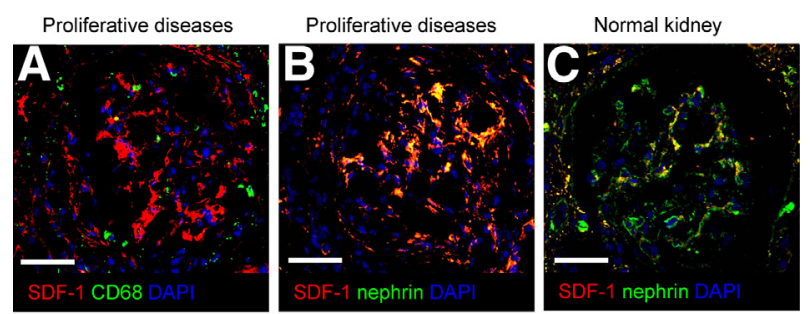

D

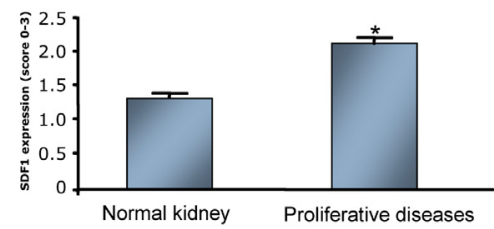

Figure 3 SDF-1 is expressed by podocytes, but not macrophages, in proliferative glomerulonephritides. A: SDF-1 (red) is highly expressed in the glomerulus of patient with extracapillary glomerulonephritis and the double immunofluorescence staining of SDF-1 (red) and CD68 (green) does not show colocalization of the two antigens. B and C: SDF-1 (red) colocalized with the podocyte marker nephrin (green). DAPI (blue) stained nuclei. Scale bars: $50 \mu \mathrm{m}$. D: SDF-1 expression is significantly increased in glomeruli of patients with extracapillary glomerulonephritis as compared with normal kidneys. ${ }^{*} P<0.05$ versus normal kidneys. 

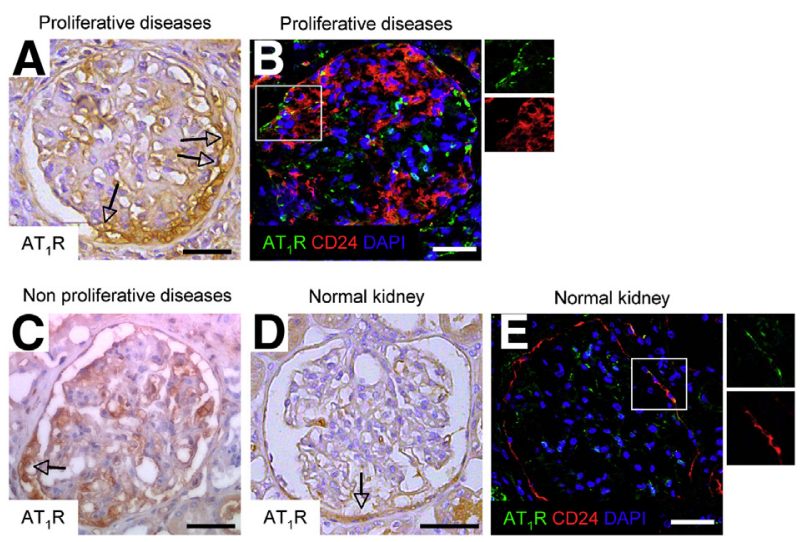

Figure $4 \quad \mathrm{AT}_{1}$ immunoexpression is up-regulated in proliferative diseases. A: Immunoperoxidase staining shows that in a patient with extracapillary glomerulonephritis, the cells forming the bridges between the Bowman's capsule and the capillary tuft abundantly express the $\mathrm{AT}_{1}$ receptor $\left(A T_{1} R\right.$; arrows). B: Double immunofluorescence staining showing some $\mathrm{CD}_{2} 4^{+}$progenitor cells (red) inside hyperplastic lesions expressing the $\mathrm{AT}_{1}$ receptor (green, right panels, which show the high magnification of the inset). C and D: Representative photomicrographs of immunoperoxidase staining in nonproliferative diseases $(\mathbf{C})$ and in a normal human glomerulus (D) showing $\mathrm{AT}_{1}$ receptor expression in a few parietal cells of the Bowman's capsule (arrows). E: Colocalization of $\mathrm{AT}_{1}$ receptor (green) and CD24 (red) expression shows that in normal human kidneys, the few parietal epithelial cells expressing the $\mathrm{AT}_{1}$ receptor are progenitor cells (right panels, which show the high magnification of the inset). DAPI (blue) stained nuclei. Scale bars: $50 \mu \mathrm{m}$.

and E) and no more abundance of ED1 macrophages than in the normal kidney (Figure 5, H and I).

\section{$\mathrm{AT}_{1}$ Receptor Overexpression on Progenitor Cells in Rat Proliferative Disorders}

We also assessed whether up-regulation and activation of the $\mathrm{AT}_{1}$ receptor on progenitor cells of the Bowman's capsule contribute to their migration and proliferation into the glomerular hyperplastic lesions. In these animals, the $\mathrm{AT}_{1}$ receptor was abundantly expressed by glomerular cells including parietal epithelial cells (Figure 6, A and D), as well as in the area of hyperplastic lesions (Figure 6A), very reminiscent of the expression profile in patients with proliferative disorders. Consistent with our previous findings,${ }^{20} \mathrm{MWF}$ rats given the ACE inhibitor lisinopril showed negligible renal progenitor cell proliferation and migration. This treatment was associated with a significant reduction in the number of progenitor cells in the Bowman's capsule expressing the $\mathrm{AT}_{1}$ receptor compared with untreated animals (Figure 6, B-D), suggesting a key role of the $\mathrm{AT}_{1}$ receptor in progenitor cell-mediated extracapillary proliferation.

\section{ACE Inhibitor Therapy Limits Progenitor Cell Proliferation by Restoring $\mathrm{AT}_{1}$ Receptor Expression in a Patient with Extracapillary Glomerulonephritis}

To provide evidence that up-regulation of $\mathrm{AT}_{1}$ receptor contributes to the formation of glomerular hyperplastic lesions in humans as well, we took advantage of the case of a 69-year-old patient with antineutrophil cytoplasm antibody-positive crescentic glomerulonephritis (already included in the cohort of patients studied here) who underwent a repeated renal biopsy after 8-month treatment with an ACE inhibitor (Figure 7A). At the first biopsy, $73.7 \%$ of the glomeruli were affected by florid extracapillary proliferation, whereas the remnant glomeruli were totally sclerotic, and no one glomerulus was intact and free of lesions (Figure 7, B and C). Because of high proteinuria (2.60 $\mathrm{g} /$ day) and severe renal insufficiency (serum creatinine, $6.99 \mathrm{mg} / \mathrm{dL}$ ), the patient received standard immunosuppressive therapy with steroids and cyclophosphamide. After 7 months of treatment, proteinuria worsened $(3.70 \mathrm{~g} / \mathrm{day})$, despite mild amelioration of renal function (serum creatinine, $4.37 \mathrm{mg} / \mathrm{dL}$ ) (Figure 7A). Thus, therapy was modified and increasing doses of the ACE inhibitor ramipril (from 2.5 to $7.5 \mathrm{mg} / \mathrm{day}$ ) in association with azathioprine were started. Eight months later, both proteinuria and serum creatinine levels were markedly reduced to $1.34 \mathrm{~g} /$ day and $1.74 \mathrm{mg} /$ $\mathrm{dL}$, respectively (Figure 7A). At this time, a second biopsy revealed that all glomeruli in the tissue specimen were
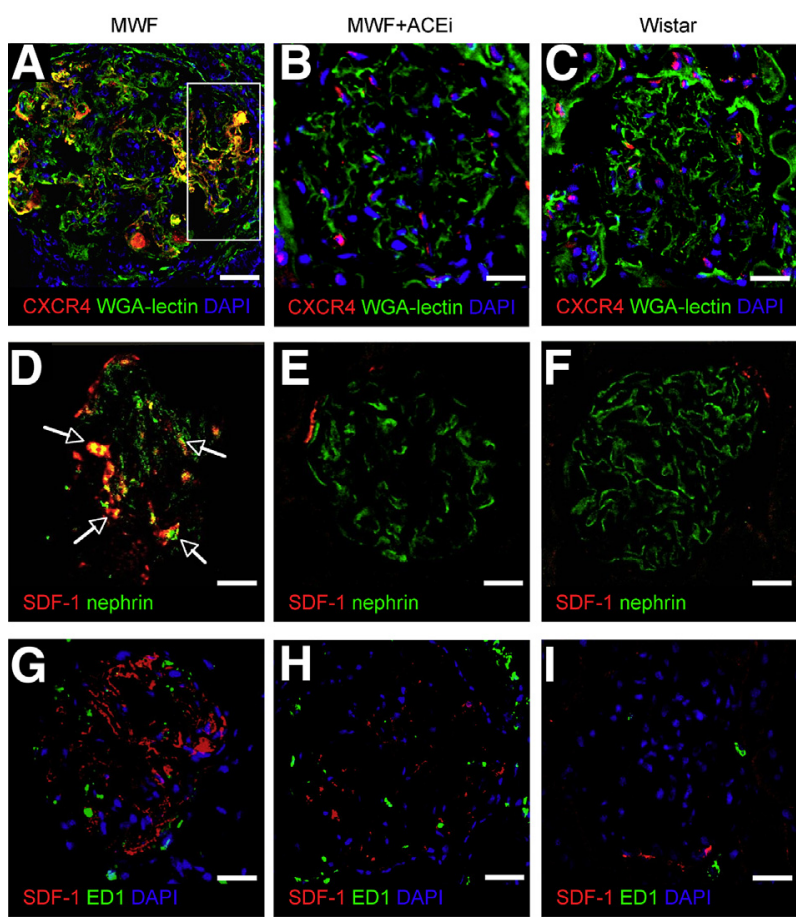

Figure 5 CXCR4 and SDF-1 up-regulation is normalized in MWF rats receiving the ACE inhibitor. A: CXCR4 (red) is markedly overexpressed by cells within hyperplastic lesions (box) in the MWF rat glomerulus. B: ACE inhibitor treatment normalizes CXCR4 expression to a level comparable with controls (C). D: SDF-1 (red) is up-regulated in the MWF rat glomerulus and colocalized with nephrin (arrows point to double-labeled podocytes stained yellow). Representative photomicrographs of double immunostaining for SDF-1 (red) and nephrin (green) (D-F) and SDF-1 (red) and ED1 (green) (G-I) show lack of association. ACE inhibitor reduces the abnormal expression of SDF-1 (red) (E and $\mathbf{H})$. DAPI (blue) stained nuclei, and renal structures were labeled with fluorescein wheat germ agglutinin (WGA; green). Scale bars: $25 \mu \mathrm{m}$. 

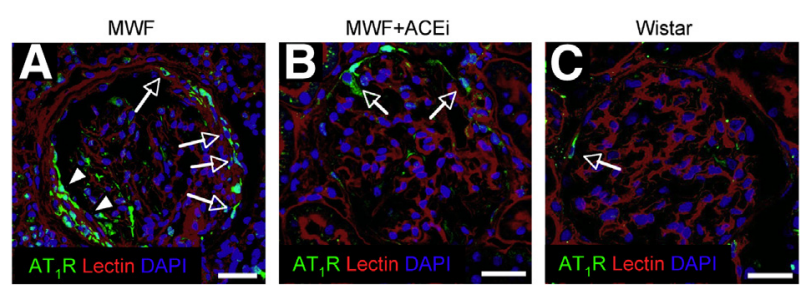

D

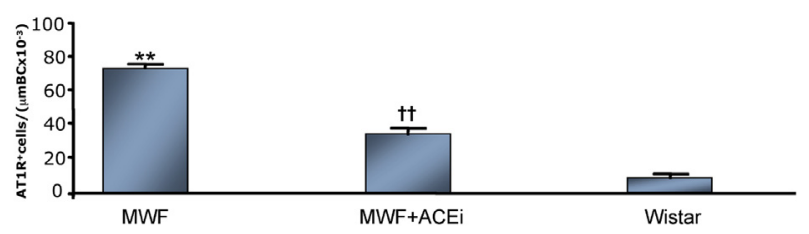

Figure $6 \quad \mathrm{AT}_{1}$ receptor overexpression in the cells of the Bowman's capsule in MWF rats is reduced by ACE inhibitor treatment. A: In MWF rats given saline, the $\mathrm{AT}_{1}$ receptor (green) is expressed by the majority of parietal epithelial cells (arrows) as well as by cells within hyperplastic lesions (arrowheads), which possibly represent renal progenitor cells. B: ACE inhibitor treatment reduces the expression of the $A T_{1}$ receptor on parietal epithelial cells (arrows). C: In Wistar rats used as controls, few cells in the Bowman's capsule expressed the $\mathrm{AT}_{1}$ receptor (arrow). DAPI (blue) stained nuclei, and renal structures were labeled with rhodamine lens culinaris agglutinin (red). Scale bars: $25 \mu \mathrm{m}$. D: The number of cells expressing the $\mathrm{AT}_{1}$ receptor along the Bowman's capsule is significantly higher in MWF rats given saline as compared with controls. $\mathrm{AT}_{1}$ receptor overexpression on parietal epithelial cells, which were assumed to be progenitors, is reduced by ACE inhibitor treatment. ${ }^{* *} P<0.01$ versus Wistar rats; ${ }^{\dagger \dagger} P<0.01$ versus MWF rats receiving saline.

totally free of crescents, suggesting the remission of crescentic lesions observed in the first biopsy (Figure 7, B-D). Overall, $38.5 \%$ of glomeruli were normal, whereas $46.1 \%$ showed global sclerosis.

Double immunostaining of the first biopsy revealed that the $\mathrm{AT}_{1}$ receptor was highly expressed and colocalized on $\mathrm{CD} 24^{+}$progenitor cells in the area of hyperplasia (Figure 7E). In the second biopsy, $\mathrm{AT}_{1}$ receptor staining was markedly reduced on $\mathrm{CD} 24^{+}$progenitor cells (Figure $7 \mathrm{~F}$ ), which were no longer present within the extracapillary space, but only localized along the Bowman's capsule (Figure 7G), as previously documented in patients with nonproliferative diseases as well as in normal kidneys. Of note, CXCR4 expression, which in the first biopsy colocalized with the progenitor cell marker CD24 in the majority of cells in crescentic lesions (Figure 2A), was only faintly expressed after ACE inhibitor treatment (Figure 7H); immunostaining being comparable to that previously observed in patients with nonproliferative nephropathies and in the normal kidney (Figures 2, B to D).

\section{Discussion}

Stemming from our recent evidence in the MWF rat model of renal disease ${ }^{20}$ and our initial study in patients with crescentic glomerulonephritis, ${ }^{19}$ here we confirmed that parietal epithelial cells expressing progenitor cell markers $\mathrm{CD} 133^{+} \mathrm{CD} 24^{+}$ proliferate and accumulate into the multilayered cellular lesions in patients with glomerulonephritides characterized by extracapillary proliferation which include extracapillary glomerulonephritis and IgA nephropathy. Moreover, in the present study, we demonstrated for the first time that the dysregulated proliferation of $\mathrm{CD} 133^{+} \mathrm{CD} 24^{+}$progenitor cells is a prominent feature of glomerulonephritides with extracapillary proliferation, but not of membranous or diabetic nephropathies. In these latter disorders, few $\mathrm{CD} 133^{+} \mathrm{CD} 24^{+}$ progenitor cells lined the inner surface of the Bowman's capsule in a manner similar to healthy human glomeruli. These findings would suggest that the abnormal proliferation of renal progenitors occurs preferentially in extracapillary glomerulonephritides, as a consequence of dysregulation of precursor cells in response to inflammatory injury and release of mediators from the glomerular tuft.

In a search of mediators responsible for the abnormal behavior of renal progenitor cells in the setting of proliferative diseases, we hypothesized a role for the chemokine SDF-1/CXCR4 axis, due to its property of promoting cell migration and proliferation. ${ }^{23,24}$ Indeed, evidence is available that the expression of CXCR4 on cancer cells correlates with the metastatic potential of multiple tumors, ${ }^{25}$ and that the interaction of CXCR4 with its ligand SDF-1 is the principal effector of hematopoietic stem cell mobilization from the bone marrow. ${ }^{23}$ Consistent with this observation are data showing that mice lacking CXCR4 or SDF-1 have a lethal defect in the colonization of bone marrow by transplanted hematopoietic stem cells. ${ }^{24}$ In addition, normal human $\mathrm{CD} 133^{+} \mathrm{CD} 24^{+}$progenitor cells have been shown to express the CXCR4 receptor. ${ }^{21}$ Based on these studies, we investigated the possible involvement of CXCR4 in the abnormal migration of $\mathrm{CD} 133^{+} \mathrm{CD} 24^{+}$progenitors in patients with glomerular proliferative diseases. Although in normal human glomeruli, only a few cells in the Bowman's capsule were positive for CXCR4, in proliferative disorders, the expression of CXCR4 was markedly up-regulated, especially in cells forming the hyperplastic lesions. Finding that such cells coexpress the stem cell marker CD24 confirms the progenitor nature of most cells in these lesions. By contrast, the faint expression of CXCR4 in cells coexpressing CD24 in membranous and diabetic nephropathies indicates that progenitor cells have little or no propensity to invade the Bowman's space in these conditions. A possible explanation of the increased CXCR4 expression on progenitor cells exclusively in proliferative diseases is offered by the inflammatory nature of these glomerular disorders. In crescentic glomerulonephritis, the general dogma is that immune complex localization in glomerular capillary wall and mesangium activates multiple humoral and cellular mediator systems including the recruitment to the glomerular tuft of strongly phlogogenic neutrophils and monocytes/macrophages. ${ }^{26,27}$ The activated cells infiltrating the glomerular tuft release soluble cytokines and chemokines that enter the Bowman's space, ${ }^{28-30}$ eventually contributing to up-regulation of adhesion molecules and chemokine receptors on parietal progenitor cells. ${ }^{5,8,9}$ In our setting, monocytes infiltrating the glomerular 

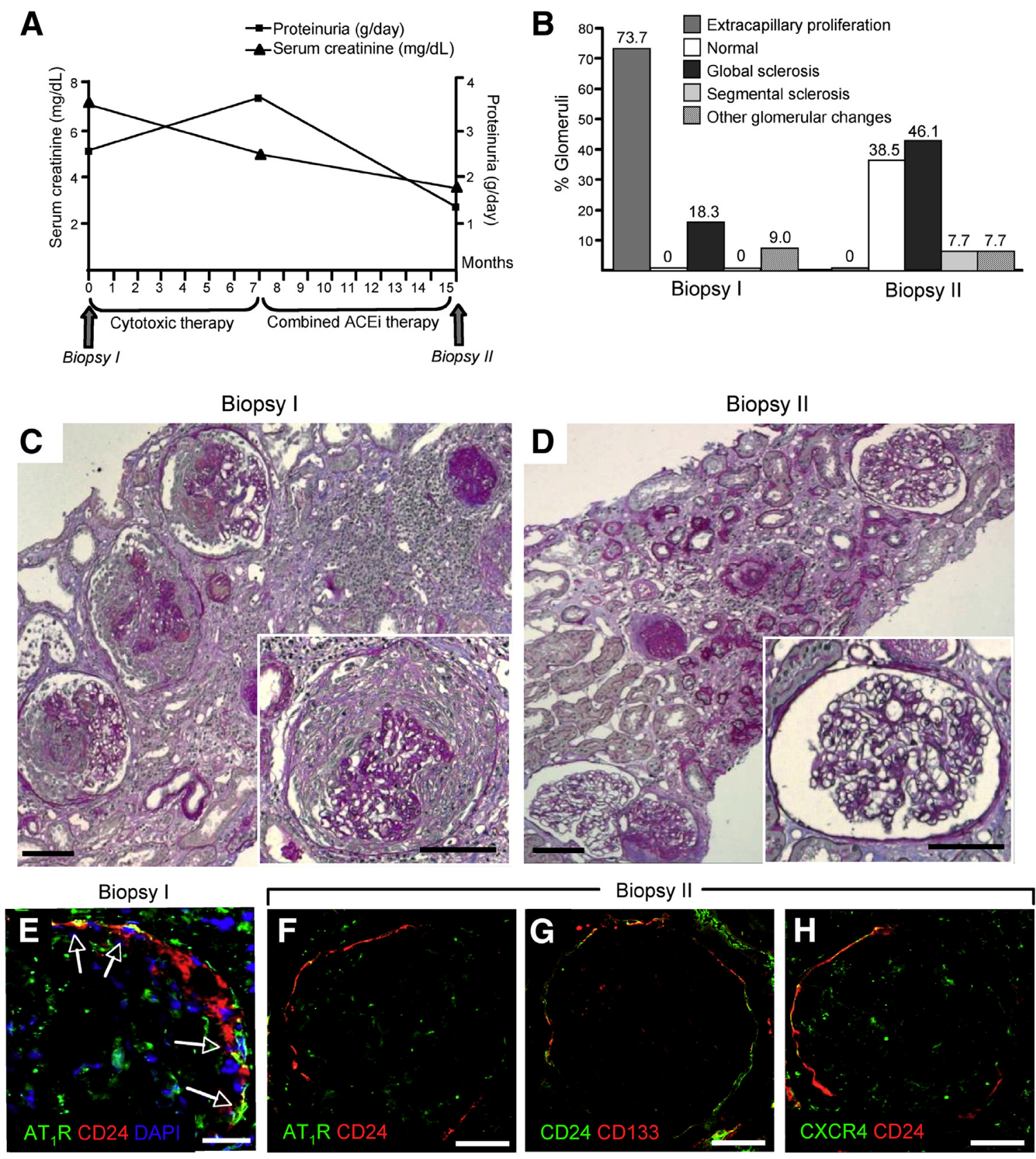

Figure $7 \quad A C E$ inhibitor ( $A C E i)$ therapy reduces progenitor cell migration associated with a normalization of $A_{1}$ receptor $\left(A_{1} T_{1}\right)$ expression in a patient with extracapillary glomerulonephritis. A: Study design and clinical findings of a patient with severe antineutrophil cytoplasm antibody-positive crescentic glomerulonephritis, who underwent renal biopsies before and after ACE inhibitor treatment. B: Histogram reporting the percentage of glomeruli with different kinds of glomerular lesions observed in renal biopsies obtained before and after ACE inhibitor treatment. C: Periodic acid-Schiff-stained biopsy of the patient before ACE inhibitor treatment reveals that the majority of glomeruli were affected by extracapillary proliferation. A representative photomicrograph showing a glomerulus with extracapillary proliferation is illustrated in the high-magnification inset. Scale bars: $100 \mu \mathrm{m}$. D: After ACE inhibitor treatment, all glomeruli of the biopsy were totally free of crescentic lesions, as shown in the representative glomerulus in the high-magnification inset. Scale bars: $100 \mu \mathrm{m}$. E: The $\mathrm{AT}_{1}$ receptor (green) was overexpressed in $\mathrm{CD}_{24}{ }^{+}$progenitor cells (red) in the area of crescent formation in the patient (arrows). Scale bar $=20 \mu \mathrm{m} . \mathrm{F}$ : After ACE inhibitor treatment, $\mathrm{AT}_{1}$ receptor (green) expression was faint, and no cells in the Bowman's capsule coexpressed the CD24 (red) progenitor marker. G: The ACE inhibitor also reduces accumulation of $\mathrm{CD}_{2} 4^{+}$(green) and $\mathrm{CD}_{133^{+}}$(red) progenitor cells that were confined to the Bowman's capsule, as previously observed in normal kidney. H: Double immunostaining for CXCR4 (green) and CD24 (red) shows that the ACE inhibitor also reduced the expression of CXCR4 in comparison to patients not receiving the treatment (Figure $2 \mathrm{~A}$ ). Scale bars: $50 \mu \mathrm{m}$.

tuft $^{31}$ did not express SDF-1, whereas podocytes activated by the inflammatory microenvironment ${ }^{32}$ produced the chemokine providing the ligand for CXCR4 receptors up-regulated on $\mathrm{CD} 133^{+} \mathrm{CD} 24^{+}$progenitor cells, ultimately allowing their migration and proliferation. These observations contribute to the knowledge of how podocyte activation could affect the behavior of parietal $\mathrm{CD} 133^{+} \mathrm{CD} 24^{+}$progenitors. Finding a similar pattern of expression of CXCR4 in activated parietal epithelial 
cells and SDF-1 in podocytes in MWF rats further substantiates our belief that the production of a chemokine (SDF-1) of podocyte origin acts as trigger for the activation of progenitor cells in the Bowman's capsule leading to hyperplasia.

Additionally, local production of Ang II, the key peptide of the renin-angiotensin system, is increased in proteinuric glomerulonephritis. ${ }^{33}$ Phlogogenic cells can release enzymes that generate Ang II, including ACE in monocytes/ macrophages ${ }^{34,35}$ and cathepsin $\mathrm{G}$ from neutrophils. ${ }^{36}$ The local accumulation of Ang II activates $\mathrm{AT}_{1}$ receptors in different cell types further sustaining the inflammatory environment via the production of reactive oxygen species, cytokines, and adhesion molecules. ${ }^{37}$ There is also evidence that Ang II is capable of up-regulating $\mathrm{AT}_{1}$ receptor expression in a dose- and time-dependent manner in macrophages. ${ }^{38}$ Likewise in hypertensive rats, chronic Ang II infusion increases $\mathrm{AT}_{1}$ receptor mRNA levels in rostral ventrolateral medulla. ${ }^{39}$ Because Ang II promotes cell migration and proliferation via $\mathrm{AT}_{1}$ receptor, ${ }^{22}$ we sought to assess whether $\mathrm{CD} 133^{+} \mathrm{CD} 24^{+}$progenitor cells contribute to the progression of hyperplastic lesions as a response to the excessive expression of this receptor on their surface. One major observation in this article was the presence of high numbers of progenitor cells located between the Bowman's capsule and the capillary tuft expressing $\mathrm{AT}_{1}$ receptors in patients with proliferative disorders. By contrast, only rare cells of the Bowman's capsule demonstrated $\mathrm{AT}_{1}$ receptor immunoreactivity in patients with membranous and diabetic nephropathies, similar to normal human glomeruli. To our knowledge, this is the first demonstration of $\mathrm{AT}_{1}$ receptor expression on progenitor cells and their up-regulation in the setting of hyperplastic lesion formation, highly suggestive of a contribution of the Ang II/AT 1 receptor pathway to the abnormal behavior of renal progenitors in proliferative diseases. Consistent with this possibility are the present findings in the MWF rats with hyperplastic glomerular lesions, where the majority of cells lining the Bowman's capsule as well as those constituting the lesions were highly expressive of the $\mathrm{AT}_{1}$ receptor, a phenomenon markedly reduced in animals treated with an ACE inhibitor that also restored the normal Bowman's capsule architecture. Our in vivo findings in rats are consistent with in vitro studies in monocytes and endothelial cells in which the ACE inhibitor ramipril suppressed Ang II/ $\mathrm{AT}_{1}$ receptor intracellular proinflammatory signaling, mainly by down-regulating $\mathrm{AT}_{1}$ receptor expression. ${ }^{40}$

Significantly, we found a patient with extracapillary glomerulonephritis in which the ACE inhibitor ramipril normalized the up-regulated expression of the $\mathrm{AT}_{1}$ receptor on $\mathrm{CD} 133^{+} \mathrm{CD} 24^{+}$renal progenitor cells concomitant with regression of glomerular hyperplasia, further suggesting that modulation of $\mathrm{AT}_{1}$ receptor expression on renal precursor cells also has a major impact on the development of glomerulonephritides in man. Moreover, a possible link between $\mathrm{AT}_{1}$ receptor and CXCR4 expression comes from the observation of a reduced accumulation of progenitor cells in the Bowman's space with negligible CXCR4 expression on their surface in the biopsy specimen collected after ACE inhibitor treatment, as well as in MWF rats treated with lisinopril. This relationship is further supported by findings that renin-angiotensin system inhibition with ACE inhibitors or $\mathrm{AT}_{1}$ receptor blockers attenuates CXCR4 mRNA expression and protein levels in the left atria in patients with chronic atrial fibrillation and mitral valve disease. ${ }^{41}$ As a caveat, although in the human study the reduction of proteinuria, the amelioration of renal function, and the dramatic improvement in renal histology were only observed after administration of ACE inhibitors, we cannot exclude a carryover effect of the immunosuppressive regimen to the favorable outcome.

In summary, we have documented that i) $\mathrm{CD} 133^{+} \mathrm{CD} 24^{+}$ progenitor cells are mostly responsible for the extracapillary lesions in patients with proliferative glomerular disease, but remain scarce in nonproliferative nephropathies; ii) both CXCR4 and $\mathrm{AT}_{1}$ receptor expression is up-regulated on renal progenitor cells that proliferate and migrate into the Bowman's capsule; iii) activated podocytes, but not infiltrating macrophages, express the CXCR4 ligand SDF-1; iv) ACE-inhibition therapy modulates renal progenitor cell proliferation and migration, restoring the normal glomerular architecture associated with both a down-regulation of $\mathrm{AT}_{1}$ receptor and CXCR4 on progenitor cells and SDF-1 on podocytes; and v) similar phenotypic changes occur in patients with proliferative glomerulonephritis and MWF rats with extracapillary lesions.

Taken together, these results provide new insights into the pathogenesis of hyperplasia in proliferative glomerulonephritis and allow us to advocate Ang II blockers as a novel therapeutic option. Targeting the Ang II/AT 1 receptor/CXCR4 pathogenic pathways might be beneficial in severe glomerular proliferative disorders that commonly lead to rapid loss of renal function requiring dialysis or transplantation.

\section{Acknowledgments}

We thank Dr. Ettore Sabadini for the collaboration in retrieving the clinical information of patients and Mauro Abbate for helpful discussion and critical supervision of the manuscript. We acknowledge the help of Manuela Passera in preparing the manuscript.

\section{References}

1. Falk R, Jennette JC, Nachman PH: Primary glomerular disease. Edited by Brenner BM. Brenner and Rector's the Kidney. 8 ed, ch 30. Philadelphia, Saunders Elsevier, 2008, pp 987-1066

2. Kambham N: Crescentic glomerulonephritis: an update on pauciimmune and anti-GBM diseases. Adv Anat Pathol 2012, 19:111-124

3. Jennette JC: Rapidly progressive crescentic glomerulonephritis. Kidney Int 2003, 63:1164-1177

4. Singh SK, Jeansson M, Quaggin SE: New insights into the pathogenesis of cellular crescents. Curr Opin Nephrol Hypertens 2011, 20: $258-262$ 
5. Boucher A, Droz D, Adafer E, Noel LH: Relationship between the integrity of Bowman's capsule and the composition of cellular crescents in human crescentic glomerulonephritis. Lab Invest 1987, 56:526-533

6. Nitta K, Horita S, Honda K, Uchida K, Watanabe T, Nihei H, Nagata M: Glomerular expression of cell-cycle-regulatory proteins in human crescentic glomerulonephritis. Virchows Arch 1999, 435:422-427

7. Jennette JC, Hipp CG: The epithelial antigen phenotype of glomerular crescent cells. Am J Clin Pathol 1986, 86:274-280

8. Ophascharoensuk V, Pippin JW, Gordon KL, Shankland SJ, Couser WG, Johnson RJ: Role of intrinsic renal cells versus infiltrating cells in glomerular crescent formation. Kidney Int 1998, 54:416-425

9. Lan HY, Nikolic-Paterson DJ, Atkins RC: Involvement of activated periglomerular leukocytes in the rupture of Bowman's capsule and glomerular crescent progression in experimental glomerulonephritis. Lab Invest 1992, 67:743-751

10. Le Hir M, Keller C, Eschmann V, Hahnel B, Hosser H, Kriz W: Podocyte bridges between the tuft and Bowman's capsule: an early event in experimental crescentic glomerulonephritis. J Am Soc Nephrol 2001, 12:2060-2071

11. Moeller MJ, Soofi A, Hartmann I, Le Hir M, Wiggins R, Kriz W, Holzman LB: Podocytes populate cellular crescents in a murine model of inflammatory glomerulonephritis. J Am Soc Nephrol 2004, 15:61-67

12. Bariety J, Bruneval P, Meyrier A, Mandet C, Hill G, Jacquot C: Podocyte involvement in human immune crescentic glomerulonephritis. Kidney Int 2005, 68:1109-1119

13. Ding M, Cui S, Li C, Jothy S, Haase V, Steer BM, Marsden PA, Pippin J, Shankland S, Rastaldi MP, Cohen CD, Kretzler M, Quaggin SE: Loss of the tumor suppressor Vhlh leads to upregulation of Cxcr4 and rapidly progressive glomerulonephritis in mice. Nat Med 2006, 12:1081-1087

14. Thorner PS, Ho M, Eremina V, Sado Y, Quaggin S: Podocytes contribute to the formation of glomerular crescents. J Am Soc Nephrol 2008, 19:495-502

15. Mundel P, Shankland SJ: Glomerular podocytes and adhesive interaction with glomerular basement membrane. Exp Nephrol 1999, 7 : $160-166$

16. Shankland SJ, Al'Douahji M: Cell cycle regulatory proteins in glomerular disease. Exp Nephrol 1999, 7:207-211

17. Kriz W, Hahnel B, Rosener S, Elger M: Long-term treatment of rats with FGF-2 results in focal segmental glomerulosclerosis. Kidney Int 1995, 48:1435-1450

18. Sagrinati C, Netti GS, Mazzinghi B, Lazzeri E, Liotta F, Frosali F, Ronconi E, Meini C, Gacci M, Squecco R, Carini M, Gesualdo L, Francini F, Maggi E, Annunziato F, Lasagni L, Serio M, Romagnani S, Romagnani P: Isolation and characterization of multipotent progenitor cells from the Bowman's capsule of adult human kidneys. J Am Soc Nephrol 2006, 17:2443-2456

19. Smeets B, Angelotti ML, Rizzo P, Dijkman H, Lazzeri E, Mooren F, Ballerini L, Parente E, Sagrinati C, Mazzinghi B, Ronconi E, Becherucci F, Benigni A, Steenbergen E, Lasagni L, Remuzzi G, Wetzels J, Romagnani P: Renal progenitor cells contribute to hyperplastic lesions of podocytopathies and crescentic glomerulonephritis. J Am Soc Nephrol 2009, 20:2593-2603

20. Benigni A, Morigi M, Rizzo P, Gagliardini E, Rota C, Abbate M, Ghezzi S, Remuzzi A, Remuzzi G: Inhibiting angiotensin-converting enzyme promotes renal repair by limiting progenitor cell proliferation and restoring the glomerular architecture. Am J Pathol 2011, 179: 628-638

21. Mazzinghi B, Ronconi E, Lazzeri E, Sagrinati C, Ballerini L, Angelotti ML, Parente E, Mancina R, Netti GS, Becherucci F, Gacci M, Carini M, Gesualdo L, Rotondi M, Maggi E, Lasagni L, Serio M, Romagnani S, Romagnani P: Essential but differential role for CXCR4 and CXCR7 in the therapeutic homing of human renal progenitor cells. J Exp Med 2008, 205:479-490

22. Kim S, Iwao H: Molecular and cellular mechanisms of angiotensin IImediated cardiovascular and renal diseases. Pharmacol Rev 2000, 52 : $11-34$
23. Lapidot T, Dar A, Kollet O: How do stem cells find their way home? Blood 2005, 106:1901-1910

24. Ratajczak MZ, Zuba-Surma E, Kucia M, Reca R, Wojakowski W, Ratajczak J: The pleiotropic effects of the SDF-1-CXCR4 axis in organogenesis, regeneration and tumorigenesis. Leukemia 2006, 20: 1915-1924

25. Reckamp KL, Strieter RM, Figlin RA: Chemokines as therapeutic targets in renal cell carcinoma. Expert Rev Anticancer Ther 2008, 8: 887-893

26. Sitprija V, Pipatanagul V, Mertowidjojo K, Boonpucknavig V, Boonpucknavig S: Pathogenesis of renal disease in leptospirosis: clinical and experimental studies. Kidney Int 1980, 17:827-836

27. Lai KN, Aarons I, Woodroffe AJ, Clarkson AR: Renal lesions in leptospirosis. Aust N Z J Med 1982, 12:276-279

28. Sitprija V, Pipantanagul V, Boonpucknavig V, Boonpucknavig S: Glomerulitis in typhoid fever. Ann Intern Med 1974, 81:210-213

29. Lambertucci JR, Godoy P, Neves J, Bambirra EA, Ferreira MD: Glomerulonephritis in Salmonella-Schistosoma mansoni association. Am J Trop Med Hyg 1988, 38:97-102

30. Indraprasit $\mathrm{S}$, Boonpucknavig $\mathrm{V}$, Boonpucknavig $\mathrm{S}$ : $\operatorname{IgA}$ nephropathy associated with enteric fever. Nephron 1985, 40:219-222

31. Sanchez-Martin L, Estecha A, Samaniego R, Sanchez-Ramon S, Vega MA, Sanchez-Mateos P: The chemokine CXCL12 regulates monocyte-macrophage differentiation and RUNX3 expression. Blood 2011, 117:88-97

32. Sayyed SG, Hagele H, Kulkarni OP, Endlich K, Segerer S, Eulberg D, Klussmann S, Anders HJ: Podocytes produce homeostatic chemokine stromal cell-derived factor-1/CXCL12, which contributes to glomerulosclerosis, podocyte loss and albuminuria in a mouse model of type 2 diabetes. Diabetologia 2009, 52:2445-2454

33. Kinoshita Y, Kondo S, Urushihara M, Suga K, Matsuura S, Takamatsu M, Shimizu M, Nishiyama A, Kawachi H, Kagami S: Angiotensin II type I receptor blockade suppresses glomerular reninangiotensin system activation, oxidative stress, and progressive glomerular injury in rat anti-glomerular basement membrane glomerulonephritis. Transl Res 2011, 158:235-248

34. Nahmod KA, Vermeulen ME, Raiden S, Salamone G, Gamberale R, Fernandez-Calotti P, Alvarez A, Nahmod V, Giordano M, Geffner JR: Control of dendritic cell differentiation by angiotensin II. FASEB J 2003, 17:491-493

35. Wassmann S, Laufs U, Baumer AT, Muller K, Konkol C, Sauer H, Bohm M, Nickenig G: Inhibition of geranylgeranylation reduces angiotensin II-mediated free radical production in vascular smooth muscle cells: involvement of angiotensin AT1 receptor expression and Rac1 GTPase. Mol Pharmacol 2001, 59:646-654

36. Sadoshima J: Cytokine actions of angiotensin II. Circ Res 2000, 86: 1187-1189

37. Brasier AR, Recinos A 3rd, Eledrisi MS: Vascular inflammation and the renin-angiotensin system. Arterioscler Thromb Vasc Biol 2002, 22 : $1257-1266$

38. Guo F, Chen XL, Wang F, Liang X, Sun YX, Wang YJ: Role of angiotensin II type 1 receptor in angiotensin II-induced cytokine production in macrophages. J Interferon Cytokine Res 2011, 31: $351-361$

39. Nunes FC, Braga VA: Chronic angiotensin II infusion modulates angiotensin II type I receptor expression in the subfornical organ and the rostral ventrolateral medulla in hypertensive rats. J Renin Angiotensin Aldosterone Syst 2011, 12:440-445

40. Schmeisser A, Soehnlein O, Illmer T, Lorenz HM, Eskafi S, Roerick O, Gabler C, Strasser R, Daniel WG, Garlichs CD: ACE inhibition lowers angiotensin II-induced chemokine expression by reduction of NF-kappaB activity and AT1 receptor expression. Biochem Biophys Res Commun 2004, 325:532-540

41. Wang XX, Zhang FR, Zhu JH, Xie XD, Chen JZ: Up-regulation of CXC chemokine receptor 4 expression in chronic atrial fibrillation patients with mitral valve disease may be attenuated by reninangiotensin system blockers. J Int Med Res 2009, 37:1145-1151 